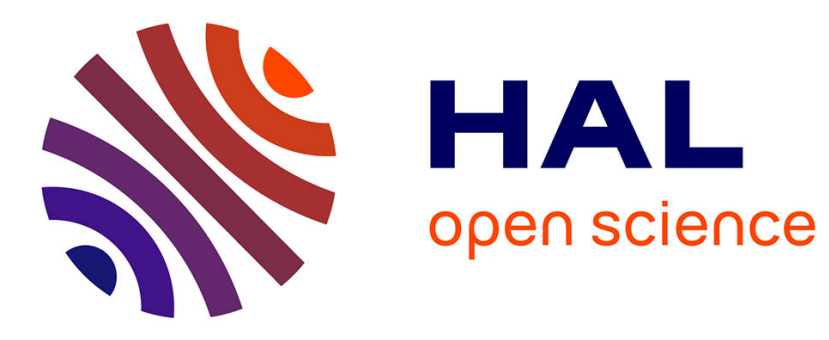

\title{
Induced agitation in homogeneous bubbly flows at moderate particle Reynolds number
}

\author{
Alain H. Cartellier, Marcello Andreotti, Philippe Séchet
}

\section{To cite this version:}

Alain H. Cartellier, Marcello Andreotti, Philippe Séchet. Induced agitation in homogeneous bubbly flows at moderate particle Reynolds number. Physical Review E: Statistical, Nonlinear, and Soft Matter Physics, 2009, 80, pp.065301. 10.1103/PhysRevE.80.065301 . hal-00643952

\section{HAL Id: hal-00643952 https://hal.science/hal-00643952}

Submitted on 3 Jan 2012

HAL is a multi-disciplinary open access archive for the deposit and dissemination of scientific research documents, whether they are published or not. The documents may come from teaching and research institutions in France or abroad, or from public or private research centers.
L'archive ouverte pluridisciplinaire $\mathbf{H A L}$, est destinée au dépôt et à la diffusion de documents scientifiques de niveau recherche, publiés ou non, émanant des établissements d'enseignement et de recherche français ou étrangers, des laboratoires publics ou privés. 


\title{
Induced agitation in homogeneous bubbly flows at moderate particle Reynolds number
}

\author{
Alain Cartellier, Marcelo Andreotti, and Philippe Sechet \\ Laboratoire des Ecoulements Géophysiques et Industriels, CNRS/UJF/Grenoble-INP, UMR 5519, BP 53, 38041 Grenoble, France
}

(Received 24 July 2009; published 17 December 2009)

\begin{abstract}
We investigated the agitation induced in a liquid by quasimonodispersed spherical bubbles. For $\operatorname{Re}_{p}$ about 10 and for void fractions ranging from 0.002 to 0.1 , the axial velocity fluctuation probability density functions happen to be uniquely determined by the velocity variance. In addition, the variance experiences a rupture from a nonlinear to a quasilinear increase with the volumic concentration. It is shown that the presence of a deficit of neighbors in the wake of any test inclusion forces the screening of velocity disturbances at a finite distance $X$ - of order $\mathrm{Re}_{p} / \alpha$ - and that the velocity variance does not depend on the system size when the later is large compared with $X$. Finally, the bubble-induced agitation happens to be much stronger for quasimonodispersed than for polydispersed size distributions because of the longer interaction duration between pairs in the former case.
\end{abstract}

DOI: 10.1103/PhysRevE.80.065301

PACS number(s): 47.55.- t, 47.57.ef

When inclusions (bubbles, droplets, and solid particles) are left to rise or fall in an inert continuous phase velocity fluctuations occur in both phases even if the base flow is laminar. Such fluctuations are exploited in a number of applications to mix phases, temperature, chemical species,.... They also give rise to the so-called pseudoturbulence stress tensor $\boldsymbol{A}=\left\langle X_{c} \boldsymbol{w}^{\prime} \boldsymbol{w}^{\prime}\right\rangle$, where $\langle\cdot\rangle$ denotes the ensemble average over all the possible positions and velocities of the inclusions, $\boldsymbol{w}^{\prime}$ is the continuous phase velocity fluctuation relative to its unconditional average $\mathbf{w}$, and $X_{c}$ is the continuous phase indicator function. In Eulerian two-fluid models, the tensor enters the unconditional momentum equation for the continuous phase as a Reynolds stress: its key role is to uniformize the spatial distribution of inclusions [1].

Yet, the origin of these fluctuations is still poorly understood. They do not reduce to streamlines distortions in a random array [2]. According to statistical two-fluid models $[3,4]$, the agitation tensor $\boldsymbol{A}$ arises from the average perturbations $\mathbf{w}^{*}\left(\boldsymbol{x} \mid \boldsymbol{x}^{\circ}\right)$ induced at a location $\boldsymbol{x}$ by a test particle centered at $\boldsymbol{x}^{\circ}$. These disturbances are summed up over all the positions $\boldsymbol{x}^{\circ}$ of the test particle, each position $\boldsymbol{x}^{\circ}$ being weighted by the corresponding number density $\phi$ :

$$
\boldsymbol{A}(\boldsymbol{x})=\int \phi\left(\boldsymbol{x}^{\circ}\right) \mathbf{w}^{*} \mathbf{w}^{*}\left(\boldsymbol{x} \mid \boldsymbol{x}^{\circ}\right) d \boldsymbol{x}^{\circ}+O\left(\alpha^{2}\right) .
$$

The average perturbed field $\mathbf{w}^{*}=\mathbf{w}\left(\boldsymbol{x} \mid \boldsymbol{x}^{\circ}\right)-\mathbf{w}(\boldsymbol{x})$ is the difference between the conditional continuous phase velocity $\mathbf{w}\left(\boldsymbol{x} \mid \boldsymbol{x}^{\circ}\right)$ at $\boldsymbol{x}$ evaluated over the subset of realizations such that an inclusion is centered at $x^{\circ}$ and the unconditional continuous phase velocity $\mathbf{w}(\boldsymbol{x})$ at $\boldsymbol{x}$. The famous Caflish and Luke argument exploits the above relationship for $\operatorname{Re}_{p}$ $=a U_{r} / \nu_{c} \ll 1\left(\nu_{c}\right.$ denotes the continuous phase kinematic viscosity, $U_{r}$ denotes the inclusion relative velocity, and $a$ denotes the inclusion radius). Assuming Stokes disturbances that slowly decay, as $1 / r$, with the distance $r$ to the test inclusion and a random suspension with uniform probability for the positions of inclusions, Caflish and Luke found that $A$ scales as $\alpha U_{r}^{2} L / a$, where $\alpha$ is the volumic concentration $\left(=4 / 3 \pi a^{3} \phi\right)$ and $L$ is the extent of the integration domain [5]. That result, which also holds for particles of finite size, implies that the agitation should linearly increase with the vessel dimension, and thus it may go to infinity. Introducing some inertia in the inclusion dynamics does not solve that issue. Indeed, for Oseen wakes and uniform probability of inclusion positions, $A$ scales as $\alpha U_{r}^{2} \ln (L / a)$, and the velocity variance is still "diverging" with the vessel size [6]. Only when potential disturbances are assumed, thus Eq. (1) leads to a finite agitation proportional to $\alpha U_{r}^{2}$. This is because of the stronger decay rate, as $r^{-3}$, of the velocity perturbation with the distance to the test inclusion $[7,8]$.The above results indicate that the induced agitation may not be liable to a local closure, in which case the resolution of two-fluid models would be drastically complexified. Yet, all these estimates rely on two key assumptions, namely, that the spatial distribution of inclusions is random (uniform probability for positions) and that the velocity disturbance is set by the dynamics of an isolated particle.

The seminal paper by Koch and Shaqfeh [3] shows how the existence of a microstructuration of the inclusions and more precisely of a particle deficit in the wake of any test inclusion can lead to the screening of the velocity disturbance at some finite distance $X$ from the test particle. In such circumstances, the induced agitation becomes finite and its magnitude is a function of the correlation length $X$ provided that the later is smaller than the system size. For a vanishing particle Reynolds number, they introduce three particles interactions (in the creeping limit, the symmetrical two-body interactions do not bring any departure from uniformity) and predict a correlation length of the order of $a / \alpha$ that leads to a velocity variance $A \propto U_{r}^{2}(\alpha X / a) \approx 4.7 U_{r}^{2}$. Although experiments performed in suspensions do not confirm that scaling because collective effects happen to drive the system behavior $[9,10]$, the proposal was nevertheless promising. It was later applied to slightly inert, polydispersed inclusions by Koch [6] who showed that the interaction of two such inclusions leads to a pair density deficit in the wake of any test inclusion the length of which $X / a=P /\left(\alpha \operatorname{Re}_{p}\right)$ increases with the width $P$ of the terminal velocity distribution (here $P$ denotes the standard deviation normalized by the mean). In that case, the agitation normalized by the square of the relative velocity, $A / U r^{2}$, was predicted to be proportional to $\alpha / \operatorname{Re}_{p}$. 


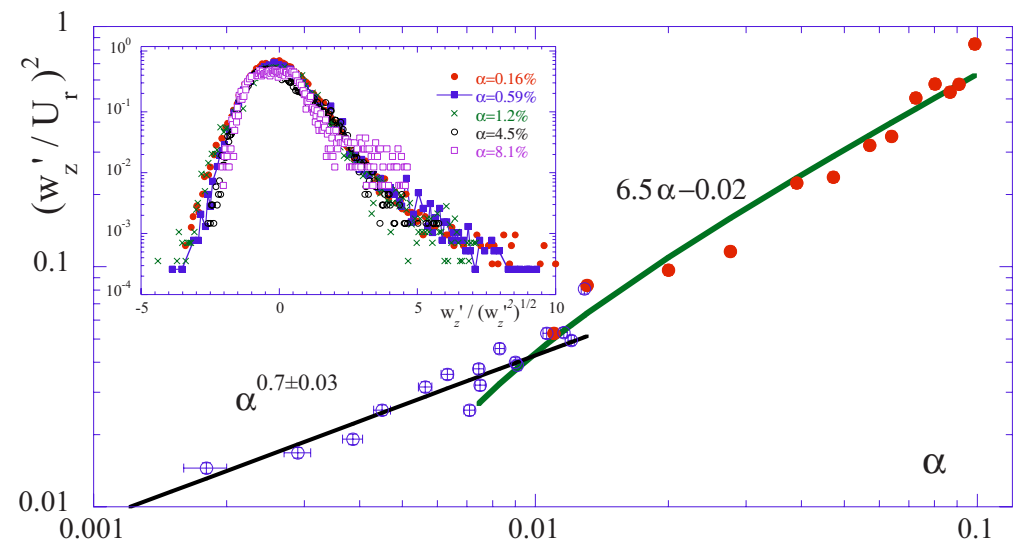

That proposal was compared with experiments performed in bubbly flows at $\operatorname{Re}_{p}$ in the range 1-50 [11]. The results were contrasted. In all the cases investigated, the velocity disturbances were screened and the induced agitation was found to be insensitive to the vessel dimension. Yet, for $\operatorname{Re}_{p}$ close to unity, the velocity variance was found to be proportional to $\alpha / \operatorname{Re}_{p}$ - as predicted by Koch-but no microstructure was detected (at least for distances to the test inclusion above $2.5-3 a$ ). At $20<\operatorname{Re}_{p}<40$ and for very dilute conditions, a neat pair density deficit was indeed observed in the wake of test bubbles, but the agitation experienced a nonlinear increase with the void fraction. Moreover, in all these conditions, the magnitude of the velocity variance was typically ten times higher than Koch's predictions (Figs. 9 and 24 in [11]).

To clarify these issues, new experiments were undertaken at a fixed particle Reynolds number, close to $10 \pm 2$, and for void fractions ranging from 0.002 to 0.1 . Bubbles were injected at the bottom of a long $(>1 \mathrm{~m})$ vertical cylindrical tube (internal diameter $40 \mathrm{~mm}$ ) through 289 injectors (internal diameter $90 \mu \mathrm{m}$ and length $20 \mathrm{~mm}$ ). In that gas-lift system, the liquid recirculation was external to that tube and took place in a large squared tank (width $350 \mathrm{~mm}$ ). The viscosity of the water-glycerine mixture was 6-8 $\times 10^{-6} \mathrm{~m}^{2} / \mathrm{s}$ so that bubbles remain spherical, with clean interfaces. The size distribution was nearly monodispersed (its standard deviation was 0.055 times the mean bubble size). The void fraction was varied by way of the gas flow rate, but this technique implies a change in the bubble diameter, in $U_{r}$, and thus in $\operatorname{Re}_{p}$. To reach high void fractions at a fixed $\mathrm{Re}_{p}$, bubbles were forced to accumulate by locating the tube exit above the free interface position at a given gas flow rate. In the center of the tube, the mean phasic flows proved to be homogeneous at a scale larger than at least 40 bubble radii. In addition, no hindering effect was noticed.

The axial velocity variance $w_{z}^{\prime 2}$ scaled by the square of the relative velocity is reported Fig. 1 versus the void fraction. For $\alpha$ above $10 \%$, large scale instabilities are triggered in the bubbly flow, and the corresponding data (not shown) no longer correspond to uniform mean flows at large scale. For void fractions in the range $0.0018-0.1$, the conditions were stable. In that range, a neat transition occurs from a nonlinear to a linear increase in the agitation with the void fraction. For $\operatorname{Re}_{p}$ about 10 that boundary is close to $\alpha_{c} \approx 1 \%$.
FIG. 1. (Color online) Evolution of the axial velocity variance in the liquid with the void fraction. $8<\operatorname{Re}_{p}<12$ (open symbols: variable gas flow rate, closed symbols: variable tube height above the free surface). Insert: probability density functions of axial velocity fluctuations scaled by the standard deviation for various concentrations.
Such a transition, here demonstrated, explains the apparent incoherencies between previous results. In particular, former data series gathered for $\operatorname{Re}_{p}$ close to 10 exhibited either linear or nonlinear growth rates with the concentration: this is because they were not collected over the same range of void fractions. Such a transition is expected to exist for others particle Reynolds numbers provided that the bubble dynamics involves inertia and laminar wakes. Indeed and although not identified by the authors, a close examination of the data of Martinez-Mercado et al. [12] indicates that a change in the scaling occurs for at least one of their data series (see experiment " $w$ - $g$ 15\%" Fig. 16 in [12]). That transition does not appear on velocity fluctuations probability density functions (PDFs) since, whatever the regime, the later collapse when normalized by the standard deviation (Fig. 1 insert). These one parameter PDFs are strongly asymmetrical because of gravity (here, positive velocities are directed upward).

In accordance with the Koch and Shaqfeh proposal, the existence of a microstructure has been sought by measuring the pair density probability $\phi^{2}\left(x, x^{\circ}\right)$. In practice, the structure function $S=\phi^{2}\left(x, x^{\circ}\right) / \phi(x) \phi\left(x^{\circ}\right)$ was derived from optical probe measurements using a technique described elsewhere [11]. To ensure the convergence of $S$, at least 100.000 bubbles were detected, the corresponding acquisition durations being comprised between 15 and $40 \mathrm{~h}$ depending on flow conditions. A Taylor-like hypothesis based on the mean

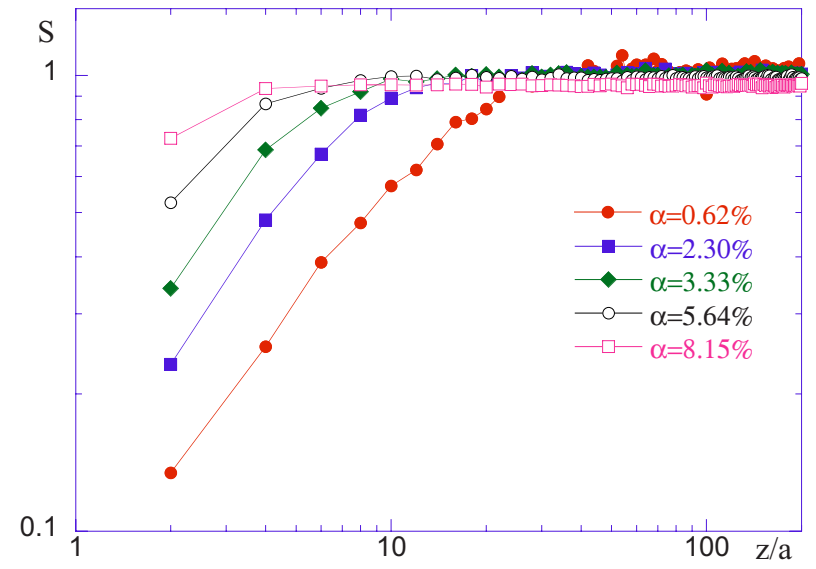

FIG. 2. (Color online) Evolution of the structure function $S$ along a vertical behind a test bubble for various void fractions. 


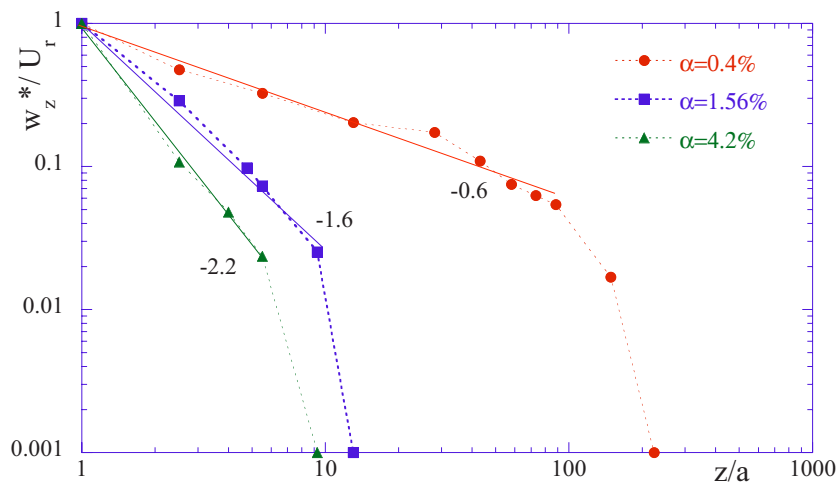

FIG. 3. (Color online) Decay of the axial component of the mean perturbed liquid velocity behind a test bubble for three void fractions.

absolute bubble velocity was used to transform time into space. Figure 2 provides the structure function along a vertical behind a test bubble. As expected, a decorrelation between bubble positions arises at large distance. At short range, $S$ is less than unity, indicating a neat deficit in the pair probability. Thus, the near wake region is almost free of neighbor inclusions. This deficit decreases with the void fraction both in magnitude and in extent. A clear microstructure is still present at a void fraction as high as $8 \%$.

To check for a possible screening of velocity disturbances, the axial component $w_{z}^{*}$ of the average disturbed velocity has been scrutinized along a vertical behind the test bubble using conditional Laser Doppler velocimetry (LDV) (Fig. 3). The Taylor hypothesis employed for $S$ was again applied to transform time into space. From the boundary condition at the interface, $w_{z}^{*}$ equals $U_{r}$ for $z=a$. Then, $w_{z}^{*}$ continuously decays with $z$, first as a power law followed by a very sharp drop. The disappearance of any velocity perturbation beyond some finite distance downstream the test inclusion is the clear manifestation of a screening effect. As the concentration increases the screening occurs at shorter range: this behavior is consistent with the strengthening of the right-hand side (rhs) terms in Eq. (2) presented thereafter.

In addition, the decay rate of $w_{z}^{*}$ with $z$ in the power-law region does not coincide with the decay rate for an isolated inclusion at the same $\mathrm{Re}_{p}$. More, the decay rate increases with the void fraction. Such deviations of the average velocity disturbance from the one due to an isolated inclusion, already reported for $\mathrm{Re}_{p}$ about 30 [11], are confirmed here for a smaller $\operatorname{Re}_{p}$. Therefore the two key hypothesis used to estimate the magnitude of the agitation and made on the velocity disturbance and on the particle spatial distribution respectively are invalidated by the present observations.

The axial extend of the deficit/screening zone has been quantified using a threshold on $S$ or on $w_{z}^{*} / U_{r}$. The results are weakly sensitive to the threshold (Fig. 4). They are also quite consistent with each over, meaning that the pair density deficit is closely linked with the extinction of velocity disturbances. The axial dimension $X$ of the deficit region is a decreasing function of $\alpha$. It experiences a transition between $\alpha^{-1 / 3}$ and $\alpha^{-1}$ behaviors (Fig. 4): the limit between these regimes corresponds to $\alpha \approx 1.5 \%$. which is close to the critical void fraction identified in Fig. 1. Thus, the change in the

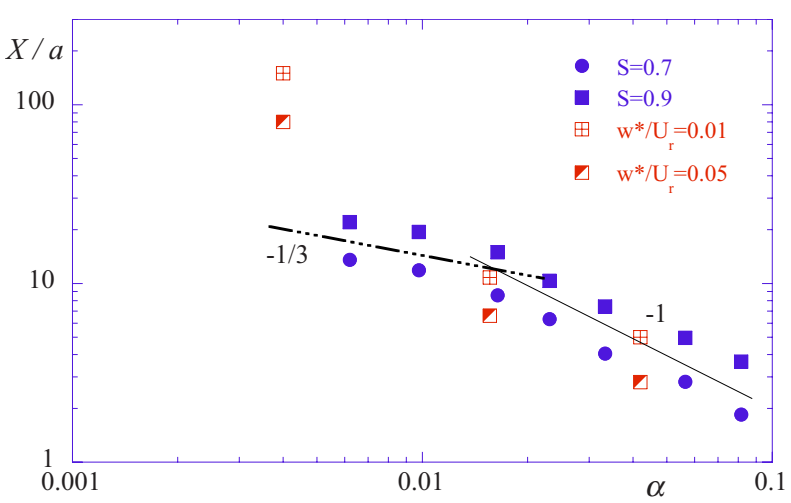

FIG. 4. (Color online) Evolution with the concentration of the vertical extension of the deficit region measured on $S$ and on $w_{z}^{*} / U_{r}$ for different thresholds.

scaling of the agitation with the concentration coincides with a modification in the scaling of the microstructure characteristic dimension with the concentration. It is worth noting that for all the conditions considered, $X$ remains less than the extent of the flow where mean variables are spatially homogeneous. This is consistent with an agitation independent of the vessel size.

Some of the above features can be recovered by considering the equations governing $\mathbf{w}^{*}\left(\boldsymbol{x} \mid \boldsymbol{x}^{\circ}\right)$. In the dilute limit, $\mathbf{w}^{*}$ is solenoidal and obeys the following momentum balance:

$$
\begin{gathered}
\rho_{c}\left\{\partial \mathbf{w}^{*} / \partial \boldsymbol{t}+\left(\mathbf{w}^{*}-\mathbf{U}_{r}\right) \cdot \partial \mathbf{w}^{*} / \partial \mathbf{x}\right\}+\partial \boldsymbol{p}^{*} / \partial \mathbf{x}-\mu_{c} \nabla^{2}\left(\mathbf{w}^{*}\right)=\phi(\mathbf{x}) \\
\times\left\{\left[1-\boldsymbol{S}\left(\mathbf{x}, \mathbf{x}^{\circ}\right)\right] \boldsymbol{F}^{*}(\mathbf{x})-\boldsymbol{S}\left(\mathbf{x}, \mathbf{x}^{\circ}\right) \boldsymbol{F}^{* *}\left(\mathbf{x} \mid \mathbf{x}^{\circ}\right)\right\},
\end{gathered}
$$

where $\rho_{c}$ (respectively, $\mu_{c}$ ) is the density (respectively the dynamic viscosity) of the continuous phase (here the liquid) and where $\mathbf{U}_{r}$ is the mean inclusion relative velocity. The source term in the right-hand side, which is zero for an isolated inclusion, is composed of two contributions. The first one is the interfacial momentum source $\phi \boldsymbol{F}^{*}$ that enters the unconditional flow momentum balance, where $\boldsymbol{F}^{*}$ is the resisting (drag) force acting on the test inclusion. The second one arises from the interfacial momentum source $\phi S\left(\boldsymbol{F}^{*}+\boldsymbol{F}^{* *}\right)$ that appears in the momentum balance governing the conditional flow $\mathbf{w}\left(\boldsymbol{x} \mid \boldsymbol{x}^{\circ}\right)$. Here, $\boldsymbol{F}^{* *}$ is the extra force experienced by the test inclusion due to the presence of another one at $\boldsymbol{x}^{\circ}$. If $S=1$, only the $\boldsymbol{F}^{* *}$ term, although small compared with $\boldsymbol{F}^{*}$, is significant. Wherever $S=0, \phi \boldsymbol{F}^{*}$ is the leading forcing term in Eq. (2). Let us introduce an idealized microstructure consisting of an axisymmetric cigarlike cell where $S=0$ while $S=1$ everywhere else (Fig. 5). Neglecting $\boldsymbol{F}^{* *}$, the force density $\phi F_{z}^{*}$ distributed in that cell counteracts the axial momentum flux in the wake of the test inclusion. Therefore the forcing progressively extinguishes the perturbed velocity field: the later disappears after some distance $X$ and no momentum escapes the cell.

The scaling for $X$ can be deduced from the balance governing the axial liquid momentum in a frame attached to the inclusion $\left(v_{z}^{*}=w_{z}^{*}-U_{r}\right)$, averaged over a cross section perpendicular to gravity. The later writes 


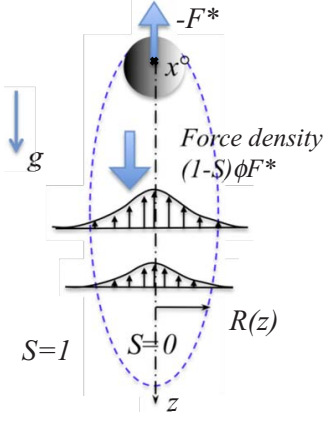

FIG. 5. (Color online) Schematic of the idealized microstructure. The force density is active within the entire cell volume.

$d / d z\left(R v_{z}^{*}\right)^{2}=\phi F_{z}^{*} R^{2} / \rho_{c}$ and its integration for a Stokes drag $F_{z}^{*}=k \pi \mu_{c} a U_{r}$ leads to

$$
v_{z}^{* 2}(z) / U_{r}^{2}=(3 k / 4)\left(\alpha / \operatorname{Re}_{p}\right) \int_{1}^{z / a} R^{2} d(z / a) / R^{2}(z) .
$$

Provided that the radial extension of the deficit zone $R(z)$ keeps the same functional form, and since the perturbed velocity drops to 0 for $z=X$, then $X / a \propto \mathrm{Re}_{p} / \alpha$ (with a prefactor about 0.015 according to the present data). Such a dependency in $\alpha$ agrees with the experiments in the domain $\alpha>\alpha_{c}$ (Fig. 4). The proportionality with $\operatorname{Re}_{p}$ is also consistent with the available results. Let us recall that no clear microstructure was detected at $\operatorname{Re}_{p}$ close to unity while $X / a$ was about 50 for $\operatorname{Re}_{p} \propto 30-50$ [11].

The above mechanism is similar to the buoyancy screening of Koch. In that two-body interaction, the trailing particle is ejected from the wake thanks to the entrainment by the perturbed velocity field $\mathbf{w}^{*}$ and to the transverse component of the force $F^{* *}$. That force arises from the interaction of the trailing particle with the perturbed field: it leads to a relative velocity $\mathbf{u}_{r}^{*}$ between that particle and the field $\mathbf{w}^{*}$. Therefore, the ejection depends on the relative velocity $\mathbf{w}^{*}+\mathbf{u}_{r}^{*}$ between the two inclusions. In monodispersed conditions, the velocity is governed by hydrodynamic interactions and not, as in Koch's model, by a difference in size or density that alters the terminal velocity. Consequently and contrary to Koch proposal, $X$ does not depend on the extent of the size distribution, and its dependence with $\operatorname{Re}_{p}$ is inversed. Monodispersed conditions imply longer pair interaction times and longer region of deficit. Since the near wake contributes the most to the induced agitation, the later happens to be much stronger in monodispersed than in polydispersed conditions. The present experiments lead indeed to $w_{z}^{\prime 2} / U_{r}^{2}=96 \alpha / \operatorname{Re}_{p}$ (for $\alpha>\alpha_{c}$ ) while Koch's predicted prefactor is $O(10)$. This large difference in magnitude can be exploited to intensify mixing processes in chemical engineering.

In the above reasoning, which applies to the domain $\alpha_{c}<\alpha$, the test-inclusion trajectory remains unaffected. We suspect that in the nonlinear region, i.e., for $\alpha<\alpha_{c}$, the lateral displacement $\Delta y$ of the test inclusion during its interaction with a rear particle becomes significant compared with the bubble size. If so, following an argument proposed by Brenner [13] for suspensions, one expects the wake to spread faster than under the action of the fluid viscosity alone. To compare $\Delta y \approx(D t)^{1 / 2}$ with $a$, the particle diffusivity $D$ is estimated as the particle velocity variance $\Delta u^{2}$ (which is close to that of the fluid [11]) multiplied by the interaction time $t \approx X / U_{r}$ (the scale of $\mathbf{w}^{*}$ is $U_{r}$ ). Thus, using the above scales, $(\Delta y / a)^{2} \approx \operatorname{Re}_{p} / \alpha$. At low $\operatorname{Re}_{p} / \alpha$, one recovers an undisturbed test-particle trajectory and a linear growth of $A$ with $\alpha$. At large $\operatorname{Re}_{p} / \alpha$, the lateral displacement is so significant that the pair density behaves as $\alpha^{-1 / 3}$ like in a random array (Fig. 4). The nonlinearity of $A$ with $\alpha$ certainly follows, but such a scaling cannot be deduced without a detailed knowledge of the disturbed velocity field. Since the later is coupled with a continuity equation governing $S$, a simulation approach is required to solve that issue. Available experiments $[11,12]$ including the present series do show that the linearity of $A$ with $\alpha$ holds for $\alpha / \operatorname{Re}_{p}>0.001-0.002$ and is lost otherwise. This behavior is fully consistent with the criterion developed here above, showing that the scaling transition we have demonstrated is controlled by the magnitude of the particle diffusity. Although extra experiments would be welcome to better define that frontier, the local closures we identified for the induced agitation at moderate particle Reynolds numbers will be quite useful to improve two-fluid models.
[1] J. L. Achard and A. Cartellier, PCH, PhysicoChem. Hydrodyn. 6, 841 (1985).

[2] A. Sangani and A. Acrivos, Int. J. Multiphase Flow 8, 343 (1982).

[3] D. Koch and E. S. G. Shaqfeh, J. Fluid Mech. 224, 275 (1991).

[4] J.-L. Achard and A. Cartellier, Arch. Mech. 53, 123 (2001).

[5] R. Caflisch and L. Luke, Phys. Fluids 28, 759 (1985).

[6] D. Koch, Phys. Fluids A 5, 1141 (1993).

[7] R. Nigmatulin, Int. J. Multiphase Flow 5, 353 (1979).
[8] A. Biesheuvel and L. Van Wijngaarden, J. Fluid Mech. 148, 301 (1984).

[9] P. N. Ségre, E. Herbolzheimer, and P. Chaikin, Phys. Rev. Lett. 79, 2574 (1997)

[10] A. Levine, S. Ramaswamy, E. Frey, and R. Bruinsma, Phys. Rev. Lett. 81, 5944 (1998).

[11] A. Cartellier and N. Rivière, Phys. Fluids 13, 2165 (2001).

[12] J. Martinez-Mercado, C. Palacios-Morales, and R. Zenit, Phys. Fluids 19, 103302 (2007).

[13] M. P. Brenner, Phys. Fluids 11, 754 (1999). 